\title{
Analysis of TRIM21 Genetic Variants on the Clinicopathologic Characteristics of Patients with Hepatocellular Carcinoma
}

\author{
Hsiang-Lin Lee ${ }^{1,2,3,+}$, Yi-Chung Chien ${ }^{4,5,6,7,8,+}$, Whei-Ling Chiang ${ }^{9}$, Hsiang-Ling Wang ${ }^{10}$, Kuan-Chun Hsueh ${ }^{2,11}$, \\ Chao-Hsuan Chen ${ }^{12}$ (D), Shuo-Chueh Chen ${ }^{13}$, Tsai-Kun Wu ${ }^{14}$, Ying-Ru Pan ${ }^{14}$, Li-Yuan Bai ${ }^{15, *}$, \\ Shun-Fa Yang $2,16, *$ (D) and Yung-Luen Yu $4,5,6,7,8,17, *$ (D)
}

\section{check for}

updates

Citation: Lee, H.-L.; Chien, Y.-C.; Chiang, W.-L.; Wang, H.-L.;

Hsueh, K.-C.; Chen, C.-H.;

Chen, S.-C.; Wu, T.-K.; Pan, Y.-R.;

Bai, L.-Y.; et al. Analysis of TRIM21

Genetic Variants on the

Clinicopathologic Characteristics of

Patients with Hepatocellular

Carcinoma. Processes 2021, 9, 495.

https://doi.org/10.3390/pr9030495

Academic Editor: Bonglee Kim

Received: 25 January 2021

Accepted: 5 March 2021

Published: 9 March 2021

Publisher's Note: MDPI stays neutral with regard to jurisdictional claims in published maps and institutional affiliations.

Copyright: (c) 2021 by the authors. Licensee MDPI, Basel, Switzerland. This article is an open access article distributed under the terms and conditions of the Creative Commons Attribution (CC BY) license (https:/ / creativecommons.org/licenses/by/ $4.0 /)$.
School of Medicine, Chung Shan Medical University, Taichung 40201, Taiwan; s31079@gmail.com Institute of Medicine, Chung Shan Medical University, Taichung 40201, Taiwan; sandersyeh@yahoo.com.tw Department of Surgery, Chung Shan Medical University Hospital, Taichung 40201, Taiwan

4 Graduate Institute of Biomedical Sciences, China Medical University, Taichung 40402, Taiwan; hardway19800710@gmail.com

5 Ph.D. Program for Translational Medicine, China Medical University, Taichung 40402, Taiwan

6 Institute of New Drug Development, China Medical University, Taichung 40402, Taiwan

7 Drug Development Center, Research Center for Cancer Biology, China Medical University, Taichung 40402, Taiwan

8 Center for Molecular Medicine, China Medical University Hospital, Taichung 40402, Taiwan

9 School of Medical Laboratory and Biotechnology, Chung Shan Medical University, Taichung 40201, Taiwan; wlchiang@csmu.edu.tw

10 Department of Beauty Science, National Taichung University of Science and Technology, Taichung 40404, Taiwan; charmaine790520@yahoo.com.tw

11 Division of General Surgery, Department of Surgery, Tungs' Taichung MetroHarbor Hospital, Taichung 43302, Taiwan

12 Department of Neurosurgery, China Medical University Hospital, Taichung 40402, Taiwan; jemileiia@yahoo.com.tw

13 Division of Pulmonary and Critical Care Medicine, Department of Internal Medicine, China Medical University Hospital, Taichung 40402, Taiwan; scchen18@gmail.com

14 Division of Renal Medicine, Tungs' Taichung MetroHarbor Hospital, Taichung 43503, Taiwan; jonesannkimo@yahoo.com.tw (T.-K.W.); liz00049@yahoo.com.tw (Y.-R.P.)

15 Division of Hematology and Oncology, Department of Internal Medicine, China Medical University Hospital, Taichung 40402, Taiwan

16 Department of Medical Research, Chung Shan Medical University Hospital, Taichung 40201, Taiwan

17 Department of Biotechnology, Asia University, Taichung 41354, Taiwan

* Correspondence: lybai6@gmail.com (L.-Y.B.); ysf@csmu.edu.tw (S.-F.Y.); ylyu@cmu.edu.tw (Y.-L.Y.)

+ These authors contributed equally to the work.

Featured Application: TRIM21 genetic variations may contribute to cancer susceptibility and distant metastasis with HCC patients.

Abstract: Tripartite motif 21 (TRIM21) plays an important role in hepatocellular carcinoma (HCC). However, the gene polymorphisms of TRIM21 in HCC is not as well known. In this study, two single nucleotide polymorphisms (SNPs) in the TRIM21 gene, rs4144331, and re915956, were selected to investigate correlations between these SNPs and susceptibility to HCC. Two SNPs of the TRIM21 gene from 1196 controls without cancer and 394 HCC patients were analyzed using realtime polymerase chain reaction. These results were further analyzed to expound the associations between these TRIM21 polymorphisms and the risk of HCC as well as the impact of these SNPs on clinicopathological characteristics of HCC. After adjustment for other covariants, we observed that that younger patients ( $<65$ years) with the TRIM21 rs915956 A allele had a probability of HCC $(\mathrm{AOR}=3.153,95 \% \mathrm{CI}: 1.315-7.516, p=0.010)$. Moreover, patients with a smoking habit who carried the T allele of rs4144331 had more probability of HCC (AOR $=2.940,95 \%$ CI: $1.331-6.491, p=0.008$ ). In addition, we observed that the polymorphic $\mathrm{T}$ allele of rs4144331 led to distant metastasis. Thus, our findings suggest that genetic variations in TRIM21 may correlate to HCC and evaluate distant metastasis in patients with HCC. 
Keywords: tripartite motif 21 (TRIM21); hepatocellular carcinoma (HCC); single nucleotide polymorphism (SNP)

\section{Introduction}

Owing to endemic hepatitis B and C virus infections, hepatocellular carcinoma (HCC) happens commonly among Asian and African populations [1]. Furthermore, HCC is one of the deadliest and most prevalent cancers worldwide. Similar to other cancers, HCC develops when mutations in the cellular machinery cause the cells to replicate at a higher rate or cause the cells to avoid undergoing apoptosis. In particular, chronic hepatitis $B$ or $C$ virus infection can cause the immune system to attack liver cells repeatedly, which contributes to the development of HCC [2]. A recent report demonstrated that the RING domain of TRIM21 can promote HBV DNA polymerase degradation. Specifically, Lys283 and Lys260 of HBV DNA Pol were identified as targets for ubiquitination mediated by TRIM21 [3]. Moreover, TRIM21 silencing has also been demonstrated to promote HCC cells [4].

Tripartite motif (TRIM) proteins are composed of multidomain ubiquitin E3 ligases with an N-terminal tripartite motif that contains three zinc-binding domains, a RING domain, a B-box type 1 and B-box type 2 domain, and a coiled coil region [5]. Consequently, TRIM proteins are considered to be key regulators of cell biology [5]. Recent studies have shown that TRIM proteins can affect cell proliferation, innate immunity, cell differentiation, cell apoptosis, and cell migration [5-11].

Like other TRIM proteins, TRIM21, also called Ro52, SSA1, or RNF81, possesses E3 ligase activity and is involved in ubiquitination [12]. Studies on TRIM21 have typically focused on its role in immunity, such as neutralizing viral infections, regulating inflammatory signaling pathways, and regulating cytokine and chemokine production [13-15]. TRIM21 was identified as an antibody-binding protein and has been shown to function as an Fc receptor that recognizes antibodies bound by intracellular pathogens. TRIM21 catalyzes the synthesis of K63 ubiquitin chains, thereby activating the innate immune system and antiviral response during pathogen invasion [16,17]. Moreover, TRIM21 inhibits the process of epithelial-mesenchymal transition (EMT) through the ubiquitination and degradation of Snail [18].

Several studies have reported on the possible involvement of genetic predisposing factors for HCC. For example, single nucleotide polymorphisms (SNPs), which are the most common type of DNA sequence variation, have shown potential for predicting cancer risk $[19,20]$. Some studies have shown that the TRIM21 gene is also associated with disease, but the number of studies is low. Recent studies have also demonstrated a possible correlation between TRIM21 allele polymorphisms and disease susceptibility as well as increased anti-TRIM21 antibodies in patients with systemic lupus erythematosus (SLE) and Sjogren syndrome (SS). Among African Americans, the rs660 C/T SNP has been shown to be related to SLE [21,22]. The rs5030767, rs5030768, rs915956, and rs4144331 SNPs were demonstrated to be associated with anti-TRIM21 antibody-positive primary SS [23]. However, the effects of TRIM21 polymorphisms on HCC are still unknown. We therefore investigated the association between TRIM21 polymorphisms and HCC. Specifically, we analyzed two SNPs, rs4144331 and rs915956, which are located in intron 3 (9571) and the $3^{\prime}$-untranslated region (3'-UTR) (12986) of the TRIM21 gene for associations among etiological, demographic, and clinical characteristics to HCC.

\section{Materials and Methods}

\subsection{Study Participants and Specimen Collection}

In this study, 394 HCC patients were recruited from Chung Shan Medical University Hospital in Taichung, Taiwan. All participants provided informed written consent during the registration process. HCC patients were clinically staged at the time of di- 
agnosis according to the tumor/node/metastasis staging system of the American Joint Committee on Cancer (AJCC, 2002). The diagnosis of cirrhosis is based on liver biopsy or abdominal ultrasound. Clinical features, including liver cirrhosis, aspartate aminotransferase (AST), the levels of $\alpha$-fetoprotein (AFP), alanine aminotransferase (ALT), tumor staging, tumor size, lymph-node metastasis, distant metastasis, presence of HBV surface antigen (HBsAg), and reactivity with antibody against HCV (anti-HCV), were collected from the chart reviews. For the control group, 1196 individuals between 20 and 70 years of age and with no history of cancer were selected from the Taiwan Biobank (https://www.twbiobank.org.tw/new_web_en/index.php, protocol code: TWBR10610-07; date of approval: 19 September 2018).

A survey concerning gender, age, cigarette smoking, and alcohol drinking was collected from each subject. An average of more than 2 drinks per day was considered as alcohol consumption. Smoking of at least one cigarette per day during the latest three months was considered a persistent smoking habit. The research was approved by the Institutional Review Board of Chung Shan Medical University Hospital.

\subsection{Selection of TRIM21 Polymorphisms}

Two SNPs in TRIM21 (NM_003141.4) were selected from the International HapMap Project data for this study. We included the SNPs rs4144331 and rs915956, which are located in intron 3 (9571) and the $3^{\prime}$-UTR (12986) region of the TRIM21 gene.

\subsection{TRIM21 Genotyping}

Allelic discrimination of the TRIM21 polymorphisms rs4144331 and rs915956 was assessed using an ABI StepOne real-time polymerase chain reaction system (Applied Biosystems, Foster City, CA, USA), SDS v3.0 software (Applied Biosystems), and the TaqMan assay [17-19].

\subsection{Statistical Analyses}

To evaluate the differences in age and demographic characteristics between the control groups and HCC patients, the Mann-Whitney U test was used. Odds ratios with 95\% confidence intervals (CIs) were estimated using logistic regression models. $p$-values of $<0.05$ were considered significant. The data were analyzed using SAS statistical software.

\section{Results}

A total of 1196 healthy controls and 394 patients with HCC were recruited for this case-cohort study. According to our analysis of the age of HCC patients (Table 1), we found significant differences in age $(p<0.001)$ between patients with HCC and healthy groups. Moreover, based on our previous study, we also found significant differences with alcohol consumption $(p<0.001), \mathrm{HBsAg}(p<0.001)$, and anti-HCV $(p<0.001)$ between patients with HCC and healthy groups [24]. These factors may tend to increase the risk of developing HCC.

Table 1. The distributions of age in 1196 controls and 394 patients with hepatocellular carcinoma (HCC).

\begin{tabular}{cccc}
\hline Variable & Controls $(\boldsymbol{n}=\mathbf{1 1 9 6})$ & Patients $(\boldsymbol{n}=\mathbf{3 9 4})$ & $\boldsymbol{p}$-Value \\
\hline Age $(\mathrm{yrs})$ & & & $p<0.001^{*}$ \\
$<65$ & $857(71.7 \%)$ & $210(53.3 \%)$ & \\
$\geqq 65$ & $339(28.3 \%)$ & $184(46.7 \%)$ & $p<0.001^{*}$ \\
Mean \pm S.D. & $59.4 \pm 7.1$ & $63.1 \pm 11.2$ & $p$
\end{tabular}

Mann-Whitney U test or Chi-square test was used between healthy controls and patients with HCC. * $p$-value $<0.05$ as statistically significant.

The distribution frequency of TRIM21 genotypes in controls and patients with HCC is shown in Table 2. No significant difference with respect to rs4144331 and rs915956 polymorphisms of TRIM21 were observed between healthy controls and patients with HCC. We further subdivided the tested population by age and found that younger patients 
( $<65$ years) with the TRIM21 rs915956 A allele t had an increased risk of HCC (AOR = 3.153, 95\% CI: 1.315-7.516, $p=0.010$; Table 3). We also subdivided the patients with HCC into smoking and nonsmoking subgroups, and found that patients with a smoking habit who carried the T allele of rs4144331 had an increased risk of HCC (AOR $=2.940,95 \%$ CI: 1.331-6.491, $p=0.008$; Table 4).

Table 2. Genotyping and allele frequency of TRIM-21 single nucleotide polymorphism (SNP) in HCC and normal controls.

\begin{tabular}{|c|c|c|c|c|}
\hline Variable & $\begin{array}{c}\text { Controls }(n=1196) \\
n(\%)\end{array}$ & $\begin{array}{c}\text { Patients }(n=394) \\
n(\%)\end{array}$ & OR (95\% CI) & $\operatorname{AOR}\left(95 \%\right.$ CI) ${ }^{a}$ \\
\hline \multicolumn{5}{|l|}{ rs4144331 } \\
\hline GG & $702(58.7 \%)$ & $231(58.6 \%)$ & 1.000 (reference) & 1.000 (reference) \\
\hline GT & $431(36 \%)$ & $133(33.8 \%)$ & $0.938(0.734-1.198)$ & $0.966(0.746-1.250)$ \\
\hline $\mathrm{TT}$ & $63(5.3 \%)$ & $30(7.6 \%)$ & $1.447(0.914-2.291)$ & $1.479(0.914-2.395)$ \\
\hline $\mathrm{GT}+\mathrm{TT}$ & $494(41.3 \%)$ & $163(41.4 \%)$ & $1.003(0.796-1.264)$ & $1.033(0.809-1.318)$ \\
\hline \multicolumn{5}{|l|}{ rs915956 } \\
\hline GG & $803(67.1 \%)$ & $273(69.3 \%)$ & 1.000 (reference) & 1.000 (reference) \\
\hline GA & $366(30.6 \%)$ & $109(27.7 \%)$ & $0.876(0.679-1.129)$ & $0.854(0.653-1.116)$ \\
\hline AA & $27(2.3 \%)$ & $12(3 \%)$ & $1.307(0.653-2.616)$ & $1.456(0.697-3.044)$ \\
\hline $\mathrm{GA}+\mathrm{AA}$ & $393(32.9 \%)$ & $121(30.7 \%)$ & $0.906(0.708-1.158)$ & $0.892(0.688-1.155)$ \\
\hline
\end{tabular}

The odds ratios (ORs) analyzed by their $95 \%$ confidence intervals (CIs) were estimated by logistic regression models. ${ }^{\text {a }}$ Adjusted for the effects of age, gender, cigarette smoking, and alcohol drinking.

Table 3. Genotyping and allele frequency of TRIM-21 single nucleotide polymorphism (SNP) among age $<65$ years old HCC and normal controls.

\begin{tabular}{|c|c|c|c|c|}
\hline Variable & $\begin{array}{c}\text { Controls }(n=857) \\
n(\%)\end{array}$ & $\begin{array}{c}\text { Patients }(n=210) \\
n(\%)\end{array}$ & OR $(95 \%$ CI) & $\operatorname{AOR}(95 \% \mathrm{CI})^{\mathrm{a}}$ \\
\hline \multicolumn{5}{|l|}{ rs4144331 } \\
\hline GG & $504(58.8 \%)$ & $120(57.1 \%)$ & 1.000 (reference) & 1.000 (reference) \\
\hline GT & $308(35.9 \%)$ & $75(35.7 \%)$ & $1.023(0.742-1.410)$ & $0.961(0.686-1.345)$ \\
\hline $\mathrm{TT}$ & $45(5.3 \%)$ & $15(7.1 \%)$ & $1.400(0.755-2.596)$ & $1.147(0.596-2.208)$ \\
\hline $\mathrm{GT}+\mathrm{TT}$ & $353(41.2 \%)$ & $90(42.9 \%)$ & $1.071(0.789-1.453)$ & $0.986(0.716-1.358)$ \\
\hline \multicolumn{5}{|l|}{ rs915956 } \\
\hline GG & $576(67.2 \%)$ & $139(66.2 \%)$ & 1.000 (reference) & 1.000 (reference) \\
\hline GA & $265(30.9 \%)$ & $62(29.5 \%)$ & $0.970(0.695-1.352)$ & $0.997(0.702-1.415)$ \\
\hline AA & $16(1.9 \%)$ & $9(4.3 \%)$ & $\begin{array}{c}2.331(1.009-5.385) \\
p=0.048\end{array}$ & $\begin{array}{c}3.153(1.315-7.561) \\
p=0.010\end{array}$ \\
\hline $\mathrm{GA}+\mathrm{AA}$ & $281(32.8 \%)$ & $71(33.8 \%)$ & $1.047(0.761-1.441)$ & $1.101(0.786-1.541)$ \\
\hline
\end{tabular}

The ORs analyzed by their $95 \%$ CIs were estimated by logistic regression models. ${ }^{\text {a }}$ Adjusted for the effects of age, gender, cigarette smoking, and alcohol drinking.

In addition, the effect of the polymorphic genotypes of TRIM21 rs4144331 on the clinical status of HCC was examined (Table 5). As shown in Table 5, patients with at least one polymorphic T allele at the rs4144331 SNP (GT + TT genotype) had a higher risk of distant metastasis (AOR $=2.773,95 \%$ CI: $1.081-7.113, p=0.034)$ and a lower risk of being infected with HCV $(p=0.048)$ compared to WT patients. Moreover, in routine blood tests conducted in conjunction with HCC diagnosis, including ALT and AST, no significant difference with respect to rs4144331 and rs915956 polymorphisms of TRIM21 was observed in patients with HCC (Table 6). 
Table 4. Genotyping and allele frequency of TRIM-21 single nucleotide polymorphism (SNP) in HCC and normal controls smokers.

\begin{tabular}{|c|c|c|c|c|}
\hline Variable & $\begin{array}{c}\text { Controls }(n=470) \\
n(\%)\end{array}$ & $\begin{array}{c}\text { Patients }(n=155) \\
n(\%)\end{array}$ & OR $(95 \%$ CI $)$ & $\operatorname{AOR}(95 \% \mathrm{CI})^{\mathrm{a}}$ \\
\hline \multicolumn{5}{|l|}{ rs4144331 } \\
\hline GG & $292(62.1 \%)$ & $92(59.4 \%)$ & 1.000 (reference) & 1.000 (reference) \\
\hline GT & $158(33.6 \%)$ & $48(31 \%)$ & $0.964(0.647-1.437)$ & $0.927(0.596-1.444)$ \\
\hline $\mathrm{TT}$ & $20(4.3 \%)$ & $15(9.7 \%)$ & $\begin{array}{c}2.380(1.171-4.838) \\
p=0.017\end{array}$ & $\begin{array}{c}2.940(1.331-6.491) \\
p=0.008\end{array}$ \\
\hline $\begin{array}{l}\mathrm{GT}+\mathrm{TT} \\
\mathrm{rs} 915956\end{array}$ & $178(37.9 \%)$ & $63(40.6 \%)$ & $1.123(0.775-1.628)$ & $1.127(0.747-1.699)$ \\
\hline GG & $306(65.1 \%)$ & $106(68.4 \%)$ & 1.000 (reference) & 1.000 (reference) \\
\hline GA & $153(32.6 \%)$ & $43(27.7 \%)$ & $0.811(0.542-1.215)$ & $0.805(0.516-1.254)$ \\
\hline AA & $11(2.3 \%)$ & $6(3.9 \%)$ & $1.575(0.568-4.362)$ & $2.076(0.653-6.604)$ \\
\hline $\mathrm{GA}+\mathrm{AA}$ & $164(34.9 \%)$ & $49(31.6 \%)$ & $0.863(0.585-1.271)$ & $0.873(0.570-1.338)$ \\
\hline
\end{tabular}

The ORs analyzed by their $95 \%$ CIs were estimated by logistic regression models. ${ }^{a}$ Adjusted for the effects of age, gender, cigarette smoking, and alcohol drinking.

Table 5. Odds ratio (OR) and 95\% confidence interval (CI) of clinical status and TRIM21 rs4144331 genotypic frequencies in HCC patients.

\begin{tabular}{|c|c|c|c|c|}
\hline \multirow[b]{2}{*}{ Variable } & \multicolumn{4}{|c|}{ Genotypic Frequencies } \\
\hline & GG $(\%)(n=231)$ & $\mathrm{GT}+\mathrm{TT}(\%)(n=163)$ & OR $(95 \% \mathrm{CI})$ & $p$ Value \\
\hline \multicolumn{5}{|l|}{ Clinical Stage } \\
\hline Stage I/II & $165(71.4 \%)$ & $110(67.5 \%)$ & 1.000 (reference) & \\
\hline Stage III/IV & $66(28.6 \%)$ & $53(32.5 \%)$ & $1.205(0.780-1.860)$ & $p=0.401$ \\
\hline \multicolumn{5}{|l|}{ Tumor size } \\
\hline$\leqq \mathrm{T} 2$ & $168(72.7 \%)$ & $112(68.7 \%)$ & 1.000 (reference) & \\
\hline$>\mathrm{T} 2$ & $63(27.3 \%)$ & $51(31.3 \%)$ & $1.214(0.782-1.885)$ & $p=0.387$ \\
\hline \multicolumn{5}{|c|}{ Lymph node metastasis } \\
\hline No & $224(97 \%)$ & $158(96.9 \%)$ & 1.000 (reference) & \\
\hline Yes & $7(3 \%)$ & $5(3.1 \%)$ & $1.013(0.316-3.248)$ & $p=0.983$ \\
\hline \multicolumn{5}{|c|}{ Distant metastasis } \\
\hline No & $224(97 \%)$ & $150(92 \%)$ & 1.000 (reference) & \\
\hline Yes & $7(3 \%)$ & $13(8 \%)$ & $2.773(1.081-7.113)$ & $p=0.034$ * \\
\hline \multicolumn{5}{|c|}{ Vascular invasion } \\
\hline No & $200(86.6 \%)$ & $130(79.8 \%)$ & 1.000 (reference) & \\
\hline Yes & $31(13.4 \%)$ & $33(20.2 \%)$ & $1.638(0.957-2.804)$ & $p=0.072$ \\
\hline \multicolumn{5}{|c|}{ Child-Pugh grade } \\
\hline $\mathrm{A}$ & $195(84.4 \%)$ & $126(77.3 \%)$ & 1.000 (reference) & \\
\hline B or $\mathrm{C}$ & $36(15.6 \%)$ & $37(22.7 \%)$ & $1.591(0.955-2.650)$ & $p=0.075$ \\
\hline \multicolumn{5}{|l|}{ HBsAg } \\
\hline Negative & $138(59.7 \%)$ & $88(54 \%)$ & 1.000 (reference) & \\
\hline Positive & $93(40.3 \%)$ & $75(46 \%)$ & $1.265(0.844-1.896)$ & $p=0.256$ \\
\hline \multicolumn{5}{|l|}{ Anti-HCV } \\
\hline Negative & $117(50.6 \%)$ & $99(60.7 \%)$ & 1.000 (reference) & \\
\hline Positive & $114(49.4 \%)$ & $64(39.3 \%)$ & $0.663(0.442-0.996)$ & $p=0.048^{*}$ \\
\hline \multicolumn{5}{|l|}{ Liver cirrhosis } \\
\hline Negative & $38(16.5 \%)$ & $30(18.4 \%)$ & 1.000 (reference) & \\
\hline Positive & $193(83.5 \%)$ & $133(81.6 \%)$ & $0.873(0.515-1.479)$ & $p=0.613$ \\
\hline
\end{tabular}

The ORs analyzed by their 95\% CIs were estimated by logistic regression models. $>$ T2: multiple tumor more than 5 cm or tumor involving a major branch of the portal or hepatic vein(s). ${ }^{*} p$-value $<0.05$ as statistically significant. 
Table 6. Association of TRIM21 genotypic frequencies with the HCC laboratory findings.

\begin{tabular}{ccccc}
\hline Characteristic & $\begin{array}{c}\alpha \text {-Fetoprotein } \\
\text { (ng/mL) }\end{array}$ & AST $^{\text {a }}$ (IU/L) & ALT $^{\text {a }}$ (IU/L) & AST/ALT $^{\text {a }}$ Ratio \\
\hline rs4144331 & & & & \\
GG & $2601.3 \pm 843.8$ & $106.4 \pm 16.3$ & $98.9 \pm 15.0$ & $1.4 \pm 0.1$ \\
GT + TT & $3834.6 \pm 1416.9$ & $126.7 \pm 22.3$ & $325.6 \pm 224.8$ & 0.968 \\
$p$ value & 0.422 & 0.979 & & 0.123 \\
rs915956 & & & $109.0 \pm 14.2$ & $1.5 \pm 0.1$ \\
GG & $2830.0 \pm 864.6$ & $126.3 \pm 18.1$ & $381.4 \pm 302.6$ & $1.4 \pm 0.1$ \\
GA + AA & $3746.6 \pm 1561.6$ & $88.9 \pm 14.0$ & 0.707 & 0.973 \\
$p$ value & 0.332 & 0.467 & \\
\hline
\end{tabular}

Mann-Whitney U test was used between two groups. ${ }^{a}$ Mean \pm S.E.

\section{Discussion}

SNPs are nucleotide variations that occur at the DNA level in every human cell. Related to environmental factors, SNPs can mimic human phenotypic diversity, but they can also indicate susceptibility to various diseases, including cancer [25]. The association between SNPs and HCC has been tested in case-controlled and prospective cohort studies based on hypothesis-driven hypothetical genetic studies. For example, many changes in hepatitis patients that affect inflammation pathways, oxidative stress, iron metabolism, or DNA repair mechanisms are related to the occurrence of liver cancer [26].

Although the relationship between TRIM21 alleles and disease susceptibility has been studied, the correlation between TRIM21 polymorphisms and risk factors in HCC has not yet been clarified. The findings of this study clarified the role of TRIM21 SNPs in HCC susceptibility and other clinicopathological conditions. The population size of rs915956 (C/C) in anti-TRIM21 antibody-positive patients with SS was approximately half of that in healthy controls [27]. From a bioinformatic analysis, it was predicted that rs4144331 may affect the binding of miRNA hsa-miR-1300. However, the detailed mechanisms of TRIM21 rs4144331 SNPs and hsa-miR-1300 in HCC cancers require future study to elucidate it.

In our previous study, we revealed that alcohol consumption was related to HCC [24]. Approximately $90 \%$ of HCC cases occur in patients with chronic liver inflammation and fibrosis due to alcohol damage [28]. According to previous studies, the results have suggested that TRIM21 acts as a tumor suppresser and is correlated with malignant potential and poor prognosis in HCC [4]. Therefore, in this study, we included the SNPs rs4144331 and rs915956 as candidate SNPs for investigating their association with HCC development. The findings showed that the presence of at least one polymorphic $\mathrm{T}$ allele of rs4144331 was strongly associated with distant metastasis (Table 5). TRIM21 can promote HBV DNA polymerase degradation with its RING domain [3]. As shown in Table 5, the association between the anti-HCV polymorphic $\mathrm{T}$ allele of rs4144331 was statistically significant. Consequently, associated with somatic genetic alterations of HCC, it is considered that constitutional SNPs may be important biomarkers for predicting response to targeted therapies, drug toxicity, and tumor recurrence after curative procedures in the future.

\section{Conclusions}

In conclusion, our findings suggest that genetic variations in TRIM21 may help to predict cancer susceptibility and distant metastasis in HCC. This study provides new information about the relationship between TRIM21 polymorphisms and the clinical pathology of HCC in the Taiwanese population.

Author Contributions: Conceptualization and data curation, H.-L.L., Y.-C.C., and S.-F.Y.; formal analysis, W.-L.C., L.-Y.B., H.-L.W., and S.-F.Y.; resources and software, H.-L.L., K.-C.H. T.-K.W., and Y.-R.P.; writing-original draft and methodology, Y.-C.C., C.-H.C., and S.-C.C.; supervision, writingreview and editing, L.-Y.B., S.-F.Y., and Y.-L.Y. All authors have read and agreed to the published version of the manuscript. 
Funding: This research was supported by grants from the Ministry of Science and Technology, Taiwan (MOST 109-2320-B-039-013-MY3), the China Medical University, Taiwan (CMU108-MF-01; CMU109MF-03), the China Medical University Hospital, Taiwan (DMR-108-BC-10; DMR-109-133; DMR110-046; DMR-110-090), the Tungs' Taichung MetroHarbor Hospital, Taiwan (TTMHH-R1100026; TTMHH-R1100027), the National Health Research Institutes, Taiwan (NHRI-110A1-CACO-13212102) and by the "Drug Development Center, China Medical University" from the Featured Areas Research Center Program within the framework of the Higher Education Sprout Project by the Ministry of Education (MOE) in Taiwan.

Institutional Review Board Statement: The study was conducted according to the guidelines of the Declaration of Helsinki, and approved by the Institutional Review Board of Chung Shan Medical University Hospital (protocol code: CSMUH No: CS17132; date of approval: 28 November 2017) and the Taiwan Biobank (protocol code: TWBR10610-07; date of approval: 19 September 2018).

Informed Consent Statement: Informed consent was obtained from all subjects involved in the study.

Conflicts of Interest: The authors declare no conflict of interest.

\section{References}

1. Huy, T.T.; Abe, K. Molecular epidemiology of hepatitis B and C virus infections in Asia. Pediatr. Int. 2004, 46, 223-230. [CrossRef]

2. Chen, C.J.; Yang, H.I.; Su, J.; Jen, C.L.; You, S.L.; Lu, S.N.; Huang, G.T.; Iloeje, U.H.; Group, R.-H.S. Risk of hepatocellular carcinoma across a biological gradient of serum hepatitis B virus DNA level. JAMA 2006, 295, 65-73. [CrossRef]

3. Mu, T.; Zhao, X.; Zhu, Y.; Fan, H.; Tang, H. The E3 Ubiquitin Ligase TRIM21 Promotes HBV DNA Polymerase Degradation. Viruses 2020, 12, 346. [CrossRef] [PubMed]

4. Ding, Q.; He, D.; He, K.; Zhang, Q.; Tang, M.; Dai, J.; Lv, H.; Wang, X.; Xiang, G.; Yu, H. Downregulation of TRIM21 contributes to hepatocellular carcinoma carcinogenesis and indicates poor prognosis of cancers. Tumour Biol. 2015, 36, 8761-8772. [CrossRef] [PubMed]

5. Ikeda, K.; Inoue, S. TRIM proteins as RING finger E3 ubiquitin ligases. Adv. Exp. Med. Biol. 2012, 770, 27-37. [CrossRef]

6. Cambiaghi, V.; Giuliani, V.; Lombardi, S.; Marinelli, C.; Toffalorio, F.; Pelicci, P.G. TRIM proteins in cancer. Adv. Exp. Med. Biol. 2012, 770, 77-91. [CrossRef] [PubMed]

7. Kawai, T.; Akira, S. Regulation of innate immune signalling pathways by the tripartite motif (TRIM) family proteins. EMBO Mol. Med. 2011, 3, 513-527. [CrossRef]

8. Petrera, F.; Meroni, G. TRIM proteins in development. Adv. Exp. Med. Biol. 2012, 770, 131-141. [CrossRef]

9. Schwamborn, J.C.; Berezikov, E.; Knoblich, J.A. The TRIM-NHL protein TRIM32 activates microRNAs and prevents self-renewal in mouse neural progenitors. Cell 2009, 136, 913-925. [CrossRef]

10. Uchil, P.D.; Pawliczek, T.; Reynolds, T.D.; Ding, S.; Hinz, A.; Munro, J.B.; Huang, F.; Floyd, R.W.; Yang, H.; Hamilton, W.L.; et al. TRIM15 is a focal adhesion protein that regulates focal adhesion disassembly. J. Cell Sci. 2014, 127, 3928-3942. [CrossRef]

11. Chang, L.C.; Yu, Y.L. Dietary components as epigenetic-regulating agents against cancer. Biomedicine (Taipei) 2016, 6, 2. [CrossRef]

12. Reddy, B.A.; van der Knaap, J.A.; Bot, A.G.; Mohd-Sarip, A.; Dekkers, D.H.; Timmermans, M.A.; Martens, J.W.; Demmers, J.A.; Verrijzer, C.P. Nucleotide biosynthetic enzyme GMP synthase is a TRIM21-controlled relay of p53 stabilization. Mol. Cell 2014, 53, 458-470. [CrossRef]

13. Kyriakidis, N.C.; Kapsogeorgou, E.K.; Gourzi, V.C.; Konsta, O.D.; Baltatzis, G.E.; Tzioufas, A.G. Toll-like receptor 3 stimulation promotes Ro52/TRIM21 synthesis and nuclear redistribution in salivary gland epithelial cells, partially via type I interferon pathway. Clin. Exp. Immunol. 2014, 178, 548-560. [CrossRef]

14. McEwan, W.A.; James, L.C. TRIM21-dependent intracellular antibody neutralization of virus infection. Prog. Mol. Biol. Transl. Sci. 2015, 129, 167-187. [CrossRef] [PubMed]

15. Young, J.A.; Sermwittayawong, D.; Kim, H.J.; Nandu, S.; An, N.; Erdjument-Bromage, H.; Tempst, P.; Coscoy, L.; Winoto, A. Fas-associated death domain (FADD) and the E3 ubiquitin-protein ligase TRIM21 interact to negatively regulate virus-induced interferon production. J. Biol. Chem. 2011, 286, 6521-6531. [CrossRef]

16. McEwan, W.A.; Tam, J.C.; Watkinson, R.E.; Bidgood, S.R.; Mallery, D.L.; James, L.C. Intracellular antibody-bound pathogens stimulate immune signaling via the Fc receptor TRIM21. Nat. Immunol. 2013, 14, 327-336. [CrossRef] [PubMed]

17. Yang, Y.S.; Yang, M.C.; Wang, B.; Weissler, J.C. Autoantigen Ro52 directly interacts with human IgG heavy chain in vivo in mammalian cells. Mol. Immunol. 2000, 37, 591-602. [CrossRef]

18. Jin, Y.; Zhang, Y.; Li, B.; Zhang, J.; Dong, Z.; Hu, X.; Wan, Y. TRIM21 mediates ubiquitination of Snail and modulates epithelial to mesenchymal transition in breast cancer cells. Int. J. Biol. Macromol. 2019, 124, 846-853. [CrossRef] [PubMed]

19. Shastry, B.S. SNP alleles in human disease and evolution. J. Hum. Genet. 2002, 47, 561-566. [CrossRef]

20. Yu, Y.L.; Su, K.J.; Hsieh, Y.H.; Lee, H.L.; Chen, T.Y.; Hsiao, P.C.; Yang, S.F. Effects of EZH2 polymorphisms on susceptibility to and pathological development of hepatocellular carcinoma. PLoS ONE 2013, 8, e74870. [CrossRef]

21. Frank, M.B.; Itoh, K.; Fujisaku, A.; Pontarotti, P.; Mattei, M.G.; Neas, B.R. The mapping of the human 52-kD Ro/SSA autoantigen gene to human chromosome 11, and its polymorphisms. Am. J. Hum. Genet. 1993, 52, 183-191. [PubMed] 
22. Tsugu, H.; Horowitz, R.; Gibson, N.; Frank, M.B. The location of a disease-associated polymorphism and genomic structure of the human 52-kDa Ro/SSA locus (SSA1). Genomics 1994, 24, 541-548. [CrossRef] [PubMed]

23. Nakken, B.; Jonsson, R.; Bolstad, A.I. Polymorphisms of the Ro52 gene associated with anti-Ro 52-kd autoantibodies in patients with primary Sjogren's syndrome. Arthritis Rheum. 2001, 44, 638-646. [CrossRef]

24. Wu, E.R.; Hsieh, M.J.; Chiang, W.L.; Hsueh, K.C.; Yang, S.F.; Su, S.C. Association of lncRNA CCAT2 and CASC8 Gene Polymorphisms with Hepatocellular Carcinoma. Int. J. Environ. Res. Public Health 2019, 16, 2833. [CrossRef] [PubMed]

25. Dong, L.M.; Potter, J.D.; White, E.; Ulrich, C.M.; Cardon, L.R.; Peters, U. Genetic susceptibility to cancer: The role of polymorphisms in candidate genes. JAMA 2008, 299, 2423-2436. [CrossRef] [PubMed]

26. Nahon, P.; Zucman-Rossi, J. Single nucleotide polymorphisms and risk of hepatocellular carcinoma in cirrhosis. J. Hepatol. 2012, 57, 663-674. [CrossRef] [PubMed]

27. Imanishi, T.; Morinobu, A.; Hayashi, N.; Kanagawa, S.; Koshiba, M.; Kondo, S.; Kumagai, S. A novel polymorphism of the SSA1 gene is associated with anti-SS-A/Ro52 autoantibody in Japanese patients with primary Sjogren's syndrome. Clin. Exp. Rheumatol. 2005, 23, 521-524. [PubMed]

28. Bishayee, A. The role of inflammation and liver cancer. Adv. Exp. Med. Biol. 2014, 816, 401-435. [CrossRef] 